\title{
ASTRALogy: Unrealistic Expectations?
}

\author{
Peter W. de Leeuw
}

Published online: 14 April 2010

(C) The Author(s) 2010. This article is published with open access at Springerlink.com

ASTRAL Investigators; Wheatley K, Ives N, Gray R, et al.: Revascularization versus medical therapy for renal-artery stenosis. N Engl J Med 2009, 361(20):1953-1962.

\section{Rating \\ -Of importance.}

\section{Introduction}

A well-recognized cause of secondary hypertension is renal artery stenosis (RAS), but uncertainty exists regarding whether it is worthwhile to actively search for this condition. First, the diagnosis of RAS requires either an invasive procedure (digital subtraction angiography [DSA]) or a noninvasive yet costly investigation such as magnetic resonance angiography (MRA) or CT angiography (CTA). One shared disadvantage of the latter two methods is that it is necessary to administer contrast material to the patient. Second, it is not so easy to select for further study those patients in whom a reasonable chance of finding a stenotic lesion exists. Finally, an effective treatment must be available in order to justify screening for RAS. Thus, starting a diagnostic strategy for RAS in a hypertensive patient makes sense only if one is prepared to take the consequences of the outcome and perform angioplasty with

P. W. de Leeuw $(\bowtie)$

Department of Medicine, University Hospital Maastricht, PO Box 5800, 6202 AZ Maastricht, The Netherlands

e-mail: p.deleeuw@intmed.unimaas.nl or without stent placement when a stenosis is considered to be hemodynamically significant. Precisely the last point is subject to debate. There are few data from randomized trials on the results of percutaneous transluminal renal angioplasty (PTRA) as compared with medical treatment in hypertensive patients with RAS. These studies were small and the results were equivocal. With respect to angioplasty plus stenting, even less information is at hand. Therefore, the Angioplasty and Stenting for Renal Artery Lesions (ASTRAL) trial was designed to explore whether angioplasty and stenting together with medical treatment would improve renal function more than medical treatment alone in patients with atherosclerotic RAS.

\begin{abstract}
Aims
ASTRAL was a multicenter, randomized, unblinded clinical trial that examined in patients with atherosclerotic RAS whether revascularization in addition to medical therapy improves renal function more than medical treatment alone.
\end{abstract}

\section{Methods}

Over 7 years, the ASTRAL investigators enrolled 806 patients from 57 hospitals (53 in the United Kingdom, 3 in Australia, and 1 in New Zealand). Patients were eligible when clinical findings such as treatment resistance or unexplained renal dysfunction suggested a diagnosis of atherosclerotic renovascular disease. When DSA, MRA, or CTA had confirmed the presence of a "substantial" stenosis in one or more renal arteries, and when the lesion was 
considered suitable for revascularization, patients were randomly assigned in a 1:1 ratio to either a group that underwent revascularization combined with medical treatment or a group receiving medical treatment only. Stratification factors during randomization were serum creatinine concentration, estimated glomerular filtration rate as derived from the Cockcroft-Gault formula [1], severity of RAS, kidney length on ultrasonography, and rate of progression of renal impairment in the year preceding enrollment into the study. Revascularization consisted of angioplasty, either alone or in combination with stent placement. Medical therapy was adjusted according to local protocols but comprised statins, antiplatelet agents, and antihypertensive drugs. After the procedure, patients were followed up at 1 to 3 months, 6 to 8 months, and 1 year. Thereafter, they were seen annually for 5 years.

Primary outcome was the change in renal function as determined from the slope of the reciprocal of serum creatinine over time. Analysis was by intention-to-treat, and the study had $80 \%$ power to detect a $20 \%$ reduction in the slope. Secondary outcomes were the level of blood pressure, the time to first renal event, the time to first major cardiovascular event, and mortality. A substudy addressed changes in cardiac structure and function, but results from this analysis are published elsewhere.

\section{Results}

The 806 patients were equally divided over both treatment groups, and there were no significant differences in baseline characteristics between the two groups. Most patients had advanced disease with over $70 \%$ stenosis or significant renal impairment. Surprisingly, though, baseline blood pressure was reasonably well controlled: on average, it was 149/ $76 \mathrm{~mm} \mathrm{Hg}$ in the revascularization group and $152 / 76 \mathrm{~mm} \mathrm{Hg}$ in the medical group. Median follow-up time was 34 months. A technically successful revascularization was achieved in 317 of the 403 patients who had been assigned to the intervention group. In $95 \%$ of these patients, angioplasty was accompanied by stent placement. In the medical-therapy group, a few patients $(6 \%)$ later underwent revascularization.

Over the 5-year follow-up period, the rate of progression of renal impairment was slightly less in the revascularization group, but the difference was small with a confidence interval crossing zero. No significant between-group differences in systolic blood pressure were apparent. Rates of renal events, major cardiovascular events, and death were similar in the two groups. Revascularization was associated with 31 serious complications in 23 patients. These included two deaths and three amputations. Post hoc analyses revealed that the overall results were similar in subgroups with varying degrees of stenosis or renal function.

\section{Discussion}

The authors conclude from their data that revascularization in patients with atherosclerotic RAS does not produce a clinically relevant benefit in terms of renal function. The same is true with respect to the secondary outcome measures such as blood pressure and renal or cardiovascular events. On the other hand, the invasive procedure was associated with substantial risk. The investigators acknowledge that the trial has some limitations, for instance with respect to the population that they studied. For ethical reasons, patients were enrolled in the trial only when their own physician was uncertain as to whether revascularization would provide a worthwhile clinical benefit. It is possible that some patients who did not meet the eligibility criteria might have benefited from revascularization.

\section{Comments}

Although the investigators do not state this explicitly, one would be inclined to interpret the results of this trial to show that it is not worthwhile, and even potentially hazardous, to perform angioplasty with or without stenting in patients with atherosclerotic RAS. The ASTRAL trial is not the first to evaluate the effects of revascularization, and I hope it will not be the last. Three previously reported randomized trials have compared angioplasty with medical treatment and have shown virtually no advantage for angioplasty. Meta-analysis of these three trials showed only a very modest improvement in blood pressure control [2], and uncertainty about this issue remained. ASTRAL is the largest randomized controlled trial so far. Unlike the previous studies, angioplasty combined with stenting (in most cases) was the revascularization procedure used in ASTRAL. In the older trials, angioplasty was not combined with stent placement. This difference makes a direct comparison difficult, as angioplasty alone carries a high risk of stenosis recurrence. Nevertheless, all four trials are consistent in the sense that the clinical benefit of revascularization is at best marginal.

This is not the end of the story, however. The patients in ASTRAL and in the other trials all had advanced atherosclerotic disease. Specifically, patients in ASTRAL were on average 70 years of age, and most had a stenosis over $70 \%$. Renal function was markedly impaired, with a mean serum creatinine level at baseline of $178 \mu \mathrm{mol} / \mathrm{L}$. This means that there was already substantial renal damage and it is a priori highly unlikely that there was much room for improvement. If we really want to do something for the kidney or, for that matter, for renovascular disease, we should probably intervene at an earlier stage, when there is still viable tissue to be salvaged. 
Disclosure No potential conflict of interest relevant to this article was reported.

Open Access This article is distributed under the terms of the Creative Commons Attribution Noncommercial License which permits any noncommercial use, distribution, and reproduction in any medium, provided the original author(s) and source are credited.

\section{References}

1. Cockcroft DW, Gault MH: Prediction of creatinine clearance from serum creatinine. Nephron 1976, 16:31-41.

2. Ives NJ, Wheatley K, Stowe RL, et al.: Continuing uncertainty about the value of percutaneous revascularization in atherosclerotic renovascular disease: a meta-analysis of randomized trials. Nephrol Dial Transplant 2003, 18:298-304. 